\title{
Synthesis, Properties and Molecular Structures of Iron(III), Cobalt(II), Nickel(II), Copper(II), Copper(I) and Zinc(II) Complexes with $N, N$-Bis(pyrazol-1-ylmethyl)benzylamine $\dagger$
}

\author{
Shiann-Cherng Sheu, Mong-Jong Tien, Ming-Chun Cheng, Tong-Ing Ho, Shie-Ming Peng* \\ and Yuan-Chuan Lin*
}

Department of Chemistry, National Taiwan University, Taipei, Taiwan, Republic of China

\begin{abstract}
A newly prepared ligand, $N, N$-bis (pyrazol-1-ylmethyl)benzylamine (L) reacted with metal ions to form $\left[\mathrm{FeLCl}_{3}\right],\left[\mathrm{NiL}(\mathrm{NCS})_{2}(\mathrm{MeOH})\right],\left[\mathrm{MLX}{ }_{2}\right]\left(\mathrm{M}=\mathrm{Co}^{\prime \prime}, \mathrm{Cu}^{\prime \prime}\right.$ or $\mathrm{Zn}^{\prime \prime}, \mathrm{X}=\mathrm{Cl}^{-}, \mathrm{Br}^{-}$or $\left.\mathrm{NCS}^{-}\right)$and $\left[(\mathrm{CuLI})_{2}\right]$. The crystallographically determined structures of $\left[\mathrm{CoLCl}_{2}\right]$ and $\left[\mathrm{CoL}(\mathrm{NCS})_{2}\right]$ reveal the co-ordination sphere of cobalt(II) to be intermediate between distorted tetrahedral and trigonal bipyramidal. In $\left[\mathrm{NiL}(\mathrm{NCS})_{2}(\mathrm{MeOH})\right]$ the co-ordination sphere of nickel(II) ion is distorted octahedral while that of copper(II) in $\left[\mathrm{CuLCl}_{2}\right]$ is distorted square pyramidal. The complex $\left[(\mathrm{CuLI})_{2}\right]$ displays distorted tetrahedral geometry around copper(1), and $\left[\mathrm{ZnLBr}_{2}\right]$ shows distorted tetrahedral geometry around zinc(II).
\end{abstract}

Polyfunctional ligands derived from pyrazole, ${ }^{1-5}$ imidazole ${ }^{6-9}$ and pyridazine ${ }^{10-12}$ form transition-metal complexes in which metal atoms are brought into proximity because of $\mathrm{N}$-donor sites that co-ordinate to metals in the systems. Structural characterizations of these molecules are vigorously pursued in many laboratories because these compounds serve as potential models for biological dimetallic sites. ${ }^{13-15}$ Some dicopper(II) complexes with pyrazolyl ligands are potential models for haemocyanin. ${ }^{16}$

In an effort to develop new polynuclear complexes, we were interested in metal complexation of polyfunctional pyrazole ligands. The previously prepared 4-methyl-2,6-bis(pyrazol-1ylmethyl)phenol ${ }^{17}$ ligand, reacted with $\mathrm{Zn}^{\mathrm{II}}$ to give a binuclear complex. 1,3,5-Tri(pyrazol-1-ylmethyl)benzene ${ }^{18}$ reacted with $\mathrm{Cu}^{\mathrm{II}}$ to give polymeric bis( $\mu$-chloro) copper(II) complexes. Other novel ligands, 1,2-bis(pyrazol-1-ylmethyl)benzene ${ }^{19}$ and 5methyl-1,3-bis(pyrazol-1-ylmethyl)benzene, ${ }^{20}$ were prepared and their abilities to complex with $\mathrm{Cu}^{\mathrm{II}}$ and $\mathrm{Co}^{\mathrm{II}}$ were reported. Here, we report an investigation of complexing properties of the pyrazolyl ligand $N, N$-bis(pyrazol-1-ylmethyl)benzylamine $\mathrm{L}$, which is a tridentate ligand with two $\mathrm{N}$-donor sites of pyrazolyl rings and one $\mathrm{N}$-donor site of benzylamine. The spectral properties and crystal structures of cobalt(II), nickel(II), copper(II), zinc(II) and copper(I) complexes of $N, N$-bis(pyrazol1 -ylmethyl)benzylamine are described.

\section{Experimental}

Chemicals.-All reagents and solvents were purchased from commercial sources and used as received unless noted otherwise. $N$-Hydroxymethylpyrazole was prepared according to the literature method. ${ }^{21}$

Physical Methods.-Melting points were obtained in open capillaries with a Thomas-Hoover capillary apparatus and are uncorrected. Proton NMR spectra were recorded on a Bruker AM-300WB instrument at $300 \mathrm{MHz}$ using $\mathrm{CDCl}_{3}$ and $\left(\mathrm{CD}_{3}\right)_{2} \mathrm{SO}$ as solvents. EPR spectra were recorded on a Bruker ESP $300 \mathrm{X}$-band instrument with diphenylpicrylhydrazyl (dpph)

† Supplementary data available: see Instructions for Authors, J. Chem. Soc., Dalton Trans., 1995, Issue 1, pp. xxv-xxx.

Non-SI unit employed: $\mu_{\mathrm{B}} \approx 9.274 \times 10^{-24} \mathrm{~J} \mathrm{~T}^{-1}$.

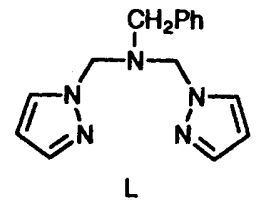

as standard. Magnetic susceptibilities were measured with the Faraday method on a CAHN 200 instrument with $\mathrm{Hg}[\mathrm{Co}-$ $\left.(\mathrm{SCN})_{4}\right]$ as standard; diamagnetic corrections were made using Pascal's parameters. Infrared spectra were recorded on a Perkin-Elmer 983G and Bomem DA8 FTIR instrument (far-IR spectra) and elemental analyses were obtained using a PerkinElmer 2400 analyser. The ultraviolet spectra were recorded on a Hitachi U-3210 spectrophotometer and solid-state electronic spectra were measured on a Shimadzu UV-2101PC UV/VIS scanning spectrophotometer $(200-800 \mathrm{~nm})$ in the diffusion reflectance mode with $\mathrm{BaO}$ as a reference.

Syntheses.-N,N-Bis(pyrazol-1-ylmethyl)benzylamine L. NHydroxymethylpyrazole $(1.96 \mathrm{~g}, 20 \mathrm{mmol})$ and benzylamine $(1.07 \mathrm{~g}, 10 \mathrm{mmol})$ in acetonitrile $\left(50 \mathrm{~cm}^{3}\right)$ were stirred in a closed vessel at room temperature for $4 \mathrm{~d}$. The acetonitrile layer was dried from the water produced by treatment with anhydrous $\mathrm{MgSO}_{4}$ and was filtered; the solvent was removed on a rotatory evaporator, yielding a slightly yellow liquid, which was used without further purification. Yield $1.92 \mathrm{~g} \mathrm{(72 \% )}$ (Found: $\mathrm{C}, 67.00 ; \mathrm{H}, 6.30 ; \mathrm{N}, 26.45$. Calc. for $\mathrm{C}_{15} \mathrm{H}_{17} \mathrm{~N}_{5}$ : C, $67.40 ; \mathrm{H}, 6.40 ; \mathrm{N}, 26.20 \%) .{ }^{1} \mathrm{H} \mathrm{NMR}\left(\mathrm{CDCl}_{3}\right): \delta 3.71(2 \mathrm{H}$, s, aryl $\left.\mathrm{CH}_{2} \mathrm{~N}\right), 4.95\left(4 \mathrm{H}, \mathrm{s}, \mathrm{CH}_{2}\right), 6.25(2 \mathrm{H}, \mathrm{t}, J 2.1$, pyrazolyl $\left.\mathrm{C}^{4} \mathrm{H}\right), 7.25-7.29(5 \mathrm{H}, \mathrm{m}$, aryl H), $7.45(2 \mathrm{H}, \mathrm{d}, J 2.1$, pyrazolyl $\left.\mathrm{C}^{5} \mathrm{H}\right)$ and $7.53\left(2 \mathrm{H}, \mathrm{d}, J 2.1 \mathrm{~Hz}\right.$, pyrazolyl $\left.\mathrm{C}^{3} \mathrm{H}\right)$. IR: $v_{\max } / \mathrm{cm}^{-1}$ $3107 \mathrm{~m}, 3029 \mathrm{~m}, 1596 \mathrm{~m}, 1507 \mathrm{~m}, 1447 \mathrm{~m}, 1390 \mathrm{~s}, 1083 \mathrm{~s}, 1044 \mathrm{~s}$, $961 \mathrm{~m}, 748 \mathrm{~s}, 699 \mathrm{~m}$ and $617 \mathrm{~m}$.

[FeLCl ${ }_{3}$ 1. Iron(III) chloride hexahydrate $(270 \mathrm{mg}, 1 \mathrm{mmol})$ in absolute ethanol $\left(15 \mathrm{~cm}^{3}\right)$ was added to a solution $\left(15 \mathrm{~cm}^{3}\right)$ of $\mathrm{L}$ in absolute ethanol $(267 \mathrm{mg}, 1 \mathrm{mmol})$. The yellow precipitate was filtered off, washed successively with ethanol and diethyl ether, and dried in air. Yield $0.36 \mathrm{~g}(82 \%)$, m.p. $158-159^{\circ} \mathrm{C}$ (decomp.) (Found: C, 41.20; H, 4.00; N, 15.70. Calc. for $\mathrm{C}_{15} \mathrm{H}_{17} \mathrm{Cl}_{3} \mathrm{FeN}_{5} \cdot 0.5 \mathrm{H}_{2} \mathrm{O}: \mathrm{C}, 41.05 ; \mathrm{H}, 4.10 ; \mathrm{N}, 15.95 \%$ ). IR: 
$v_{\max } / \mathrm{cm}^{-1} 3414(\mathrm{br}), 3130 \mathrm{~m}, 1517 \mathrm{~m}, 1456 \mathrm{~m}, 1400 \mathrm{~s}, 1281 \mathrm{~s}, 1068 \mathrm{~s}$, $993 \mathrm{~m}, 769 \mathrm{~m}, 702 \mathrm{~m}$ and $606 \mathrm{~m} . \lambda_{\max } / \mathrm{nm}(\mathrm{MeOH}) 355(\varepsilon 3406$ $\left.\mathrm{dm}^{3} \mathrm{~mol}^{-1} \mathrm{~cm}^{-1}\right), 252$ (5468) and 209 (22 336).

$\left[\mathrm{CoLCl}_{2}\right]$ 2. Cobalt(II) chloride hexahydrate $(238 \mathrm{mg}, 1$ mmol) in methanol $\left(15 \mathrm{~cm}^{3}\right)$ was added to a methanolic solution $\left(10 \mathrm{~cm}^{3}\right)$ of $\mathrm{L}(267 \mathrm{mg}, 1 \mathrm{mmol})$. The mixture was stirred for 10 min at room temperature and filtered. The blue crystalline product was obtained by slow diffusion of diethyl ether into the mixture. M.p. $180-181^{\circ} \mathrm{C}$ (Found: C, 45.60; H, 4.10; N, 17.20. Calc. for $\mathrm{C}_{15} \mathrm{H}_{17} \mathrm{Cl}_{2} \mathrm{CoN}_{5}$ : C, 45.35; $\mathrm{H}, 4.30 ; \mathrm{N}, 17.65 \%$ ). IR: $v_{\max } / \mathrm{cm}^{-1} 3440$ (br), $3122 \mathrm{~m}, 1507 \mathrm{~m}, 1448 \mathrm{~m}, 1398 \mathrm{~s}, 1354 \mathrm{~m}$, $1277 \mathrm{~m}, 1206 \mathrm{~m}, 1091 \mathrm{~m}, 1062 \mathrm{~s}, 980 \mathrm{~m}, 772 \mathrm{~s}, 754 \mathrm{~s}$ and $645 \mathrm{~s}$. $\lambda_{\text {max }} / \mathrm{nm}(\mathrm{MeOH}) 258\left(\varepsilon 342 \mathrm{dm}^{3} \mathrm{~mol}^{-1} \mathrm{~cm}^{-1}\right)$ and $209(12497)$.

[CoL(NCS) $)_{2}$ 3. Cobalt(II) nitrate hexahydrate $(291 \mathrm{mg}, 1$ $\mathrm{mmol})$ in methanol $\left(15 \mathrm{~cm}^{3}\right)$ was added to a methanolic solution $\left(10 \mathrm{~cm}^{3}\right)$ of $\mathrm{L}(267 \mathrm{mg}, 1 \mathrm{mmol})$ and a methanolic solution (10 $\left.\mathrm{cm}^{3}\right)$ of NaSCN (162 mg, $2 \mathrm{mmol}$ ) was added. The mixture was allowed to stand at room temperature. A violet crystalline product was filtered off, washed successively with methanol and diethyl ether and dried in air. Yield $0.27 \mathrm{~g}(61 \%)$. M.p. 186$187^{\circ} \mathrm{C}$ (decomp.) (Found: C, 45.35; H, 3.80; N, 21.80. Calc. for $\mathrm{C}_{17} \mathrm{H}_{17} \mathrm{CoN}_{7} \mathrm{~S}_{2} \cdot 0.5 \mathrm{H}_{2} \mathrm{O}: \mathrm{C}, 45.25 ; \mathrm{H}, 4.00 ; \mathrm{N}, 21.75 \%$ ). IR: $v_{\text {max }} / \mathrm{cm}^{-1} 3123 \mathrm{~m}, 2077 \mathrm{~s}, 2060 \mathrm{~s}, 1519 \mathrm{~m}, 1400 \mathrm{~m}, 1278 \mathrm{~m}, 1066 \mathrm{~m}$ and $764 \mathrm{~m} . \lambda_{\max } / \mathrm{nm}(\mathrm{MeCN}) 579\left(\varepsilon 705 \mathrm{dm}^{3} \mathrm{~mol}^{-1} \mathrm{~cm}^{-1}\right), 323$ (5425) and 225 (11 572)

$\left[\mathrm{NiL}(\mathrm{NCS})_{2}(\mathrm{MeOH})\right]$ 4. The complex was prepared as described for complex 3 . A blue crystalline product was filtered off, washed successively with methanol and diethyl ether and dried in air. Yield $0.32 \mathrm{~g}(68 \%)$. M.p. $199-200^{\circ} \mathrm{C}$ (decomp.) (Found: C, 45.60; H, 4.50; N, 19.85. Calc. for $\mathrm{C}_{18} \mathrm{H}_{21} \mathrm{~N}_{7} \mathrm{NiOS}_{2}$ : C, 45.60; H, 4.45; N, 20.70\%). IR: $v_{\max } / \mathrm{cm}^{-1} 3334$ (br), $3112 \mathrm{~m}$, $2088 \mathrm{~s}, 1519 \mathrm{~m}, 1446 \mathrm{~m}, 1397 \mathrm{~m}, 1273 \mathrm{~m}, 1202 \mathrm{~m}, 1093 \mathrm{~m}, 1056 \mathrm{~m}$, $977 \mathrm{~m}, 773 \mathrm{~m}, 744 \mathrm{~m}, 702 \mathrm{~m}$ and $651 \mathrm{~m} . \lambda_{\text {max }} / \mathrm{nm}(\mathrm{MeCN}) 294$ ( $\varepsilon 1332 \mathrm{dm}^{3} \mathrm{~mol}^{-1} \mathrm{~cm}^{-1}$ ) and 219 (12 284).

$\left[\mathrm{CuLCl}_{2}\right]$ 5. The complex was prepared as described for complex 2. M.p. $155-156{ }^{\circ} \mathrm{C}$ (Found: C, 44.85; H, 4.15; N, 17.55. Calc. for $\mathrm{C}_{15} \mathrm{H}_{17} \mathrm{Cl}_{2} \mathrm{CuN}_{5}: \mathrm{C}, 44.85 ; \mathrm{H}, 4.25 ; \mathrm{N}$, $17.45 \%$ ). IR: $v_{\max } / \mathrm{cm}^{-1} 3443(\mathrm{br}), 3125 \mathrm{~m}, 1507 \mathrm{~m}, 1461 \mathrm{~m}, 1410 \mathrm{~s}$, $1397 \mathrm{~s}, 1270 \mathrm{~s}, 1202 \mathrm{~m}, 1140 \mathrm{~m}, 1093 \mathrm{~m}, 1065 \mathrm{~s}, 978 \mathrm{~m}, 763 \mathrm{~m}, 737 \mathrm{~s}$, $703 \mathrm{~s}$ and $645 \mathrm{~m} . \lambda_{\max } / \mathrm{nm}(\mathrm{MeOH}) 718\left(\varepsilon 95 \mathrm{dm}^{3} \mathrm{~mol}^{-1} \mathrm{~cm}^{-1}\right)$, 265 (3397) and 209 (18 994).

$\left[(\mathrm{CuLI})_{2}\right]$ 6. Copper(I) iodide $(190 \mathrm{mg}, 1 \mathrm{mmol})$ in acetonitrile $\left(20 \mathrm{~cm}^{3}\right)$ was bubbled with $\mathrm{N}_{2}$ for $10 \mathrm{~min}$ and added to an acetonitrile solution $\left(20 \mathrm{~cm}^{3}\right)$ of $\mathrm{L}(267 \mathrm{mg}, 1 \mathrm{mmol})$. The mixture was allowed to stand at room temperature. Colourless crystals were filtered off, washed successively with acetonitrile and diethyl ether and dried in air. Yield $0.34 \mathrm{~g}(37 \%)$. M.p. 159$160^{\circ} \mathrm{C}$ (decomp.) [Found: C, 39.35; H, 3.55; N, 15.10. Calc. for $\left.\left(\mathrm{C}_{15} \mathrm{H}_{17} \mathrm{CuIN}_{5}\right)_{2}: \mathrm{C}, 39.35 ; \mathrm{H}, 3.70 ; \mathrm{N}, 15.30 \%\right]$. IR: $v_{\max } / \mathrm{cm}^{-1}$ $3104 \mathrm{~m}, 1508 \mathrm{~m}, 1392 \mathrm{~m}, 1374 \mathrm{~m}, 1241 \mathrm{~s}, 1150 \mathrm{~s}, 1100 \mathrm{~m}, 1085 \mathrm{~s}$, $1053 \mathrm{~s}, 981 \mathrm{~m}, 962 \mathrm{~m}$ and $748 \mathrm{~s} .{ }^{1} \mathrm{H}$ NMR $\left[\left(\mathrm{CD}_{3}\right)_{2} \mathrm{SO}\right]: \delta 3.84$ $(2 \mathrm{H}, \mathrm{s}), 5.20(4 \mathrm{H}, \mathrm{s}), 6.44(2 \mathrm{H}, \mathrm{s}), 7.28-7.38(5 \mathrm{H}, \mathrm{m}), 7.75$ $(2 \mathrm{H}, \mathrm{s})$ and $7.88(2 \mathrm{H}, \mathrm{s})$

$\left[\mathrm{ZnLBr}_{2}\right] 7$ and $\left[\mathrm{ZnL}(\mathrm{NCS})_{2}\right]$ 8. These complexes were prepared as described for complex 3 , above, with appropriate anions substituted for $\mathrm{Br}^{-}$and $\mathrm{NCS}^{-}$. Colourless crystals were filtered, washed successively with methanol and diethyl ether and dried in air. Yield $0.19 \mathrm{~g}(39 \%)$ for complex 7 (m.p. 176$178^{\circ} \mathrm{C}$ ) and $0.42 \mathrm{~g}(92 \%)$ for complex 8 (m.p. $149-151^{\circ} \mathrm{C}$ ) [Found: C, 36.45; H, 3.4; N, 14.65. Calc. for $\mathrm{C}_{15} \mathrm{H}_{17} \mathrm{Br}_{2} \mathrm{~N}_{5} \mathrm{Zn}$ (7): C, $36.60 ; \mathrm{H}, 3.50 ; \mathrm{N}, 14.20 \%$ ]. IR: $v_{\max } / \mathrm{cm}^{-1} 3105 \mathrm{~m}, 1523 \mathrm{~m}$, $1407 \mathrm{~m}, 1381 \mathrm{~m}, 1259 \mathrm{~m}, 1150 \mathrm{~m}, 1072 \mathrm{~s}, 986 \mathrm{~m}, 781 \mathrm{~m}, 755 \mathrm{~m}$ and $612 \mathrm{~m} .{ }^{1} \mathrm{H}$ NMR $\left[\left(\mathrm{CD}_{3}\right)_{2} \mathrm{SO}\right]: \delta 3.72(2 \mathrm{H}, \mathrm{s}), 5.07(4 \mathrm{H}, \mathrm{s}), 6.31$ $(2 \mathrm{H}, \mathrm{s}), 7.20-7.33(5 \mathrm{H}, \mathrm{m}), 7.54(2 \mathrm{H}, \mathrm{s})$ and $7.76(2 \mathrm{H}, \mathrm{s})$. $\lambda_{\max } / \mathrm{nm}(\mathrm{MeCN}) 342\left(\varepsilon 190 \mathrm{dm}^{3} \mathrm{~mol}^{-1} \mathrm{~cm}^{-1}\right)$ and $212(7334)$ [Found: C, 45.45; H, 3.75; N, 22.20. Calc. for $\mathrm{C}_{17} \mathrm{H}_{17} \mathrm{~N}_{7} \mathrm{~S}_{2} \mathrm{Zn}$ (8): C, 45.50; H, 3.80; N, 21.85\%]. IR: $v_{\max } / \mathrm{cm}^{-1} 3092 \mathrm{~m}, 2083 \mathrm{~s}$, $1523 \mathrm{~m}, 1404 \mathrm{~m}, 1065 \mathrm{~m}, 766 \mathrm{~m}, 706 \mathrm{~m}$ and $614 \mathrm{~m} .{ }^{1} \mathrm{H}$ NMR $\left[\left(\mathrm{CD}_{3}\right)_{2} \mathrm{SO}\right]: \delta 3.71(2 \mathrm{H}, \mathrm{s}), 5.05(4 \mathrm{H}, \mathrm{s}), 6.31(2 \mathrm{H}, \mathrm{s}), 7.22$ $7.32(5 \mathrm{H}, \mathrm{m}), 7.53(2 \mathrm{H}, \mathrm{s})$ and $7.76(2 \mathrm{H}, \mathrm{s}) . \lambda_{\text {max }} / \mathrm{nm}(\mathrm{MeCN})$ $342\left(\varepsilon 123 \mathrm{dm}^{3} \mathrm{~mol}^{-1} \mathrm{~cm}^{-1}\right)$ and $218(7928)$.
$\left[\mathrm{ZnLCl}_{2}\right]$ 9. Zinc(II) chloride (136 $\left.\mathrm{mg}, 1 \mathrm{mmol}\right)$ in methanol $\left(15 \mathrm{~cm}^{3}\right)$ was added to a solution of $\mathrm{L}(267 \mathrm{mg}, 1 \mathrm{mmol})$ in methanol $\left(15 \mathrm{~cm}^{3}\right)$. The mixture was allowed to stand at room temperature. Colourless crystals were filtered off, washed successively with methanol and diethyl ether and dried in air. Yield $0.36 \mathrm{~g}\left(89 \%\right.$ ). M.p. $191-192^{\circ} \mathrm{C}$ (Found: C, 44.80; H, 4.05; $\mathrm{N}, 17.00$. Calc. for $\mathrm{C}_{15} \mathrm{H}_{17} \mathrm{Cl}_{2} \mathrm{~N}_{5} \mathrm{Zn}: \mathrm{C}, 44.65 ; \mathrm{H}, 4.25 ; \mathrm{N}$, $17.35 \%$ ). IR: $v_{\max } / \mathrm{cm}^{-1} 3104 \mathrm{~m}, 1507 \mathrm{~m}, 1402 \mathrm{~s}, 1379 \mathrm{~m}, 1301 \mathrm{~m}$, $1259 \mathrm{~s}, 1149 \mathrm{~s}, 1100 \mathrm{~m}, 1073 \mathrm{~s}, 986 \mathrm{~s}, 931 \mathrm{~m}, 782 \mathrm{~s}$ and $757 \mathrm{~m} .{ }^{1} \mathrm{H}$ NMR [( $\left.\left(\mathrm{CD}_{3}\right)_{2} \mathrm{SO}\right]: \delta 3.72(2 \mathrm{H}, \mathrm{s}), 5.09(4 \mathrm{H}, \mathrm{s}), 6.31(2 \mathrm{H}, \mathrm{d})$, $7.20-7.30(5 \mathrm{H}, \mathrm{m}), 7.55(2 \mathrm{H}, \mathrm{d})$ and $7.77(2 \mathrm{H}, \mathrm{d})$. $\lambda_{\max } / \mathrm{nm}(\mathrm{MeOH}) 258\left(\varepsilon 400 \mathrm{dm}^{3} \mathrm{~mol}^{-1} \mathrm{~cm}^{-1}\right)$ and $208(11550)$.

Structural Determinations. - Suitable crystals of complexes 2-7 were chosen. Intensity data were collected at room temperature on a CAD-4 diffractometer using monochromatic Mo-K $\alpha$ radiation $(\lambda 0.7107 \AA$ ). The unit-cell parameters were derived from a least-squares refinement of 25 setting reflections for complexes 5 and 7 and 24 setting reflections for complexes 2-4 and 6. The $\theta-2 \theta$ scan technique and a variable scan speed were used to obtain integrated intensities. Three reference reflections were monitored throughout the measurement; the decay of the intensities was less than $2 \%$ in all cases. Absorption corrections were applied according to an experimental $\psi$ rotational curve. Other details of crystal data are given in Table 1 .

The structures were solved by the heavy-atom method; subsequent Fourier syntheses based on the heavy-atoms revealed the positions of all non-hydrogen atoms. Leastsquares refinement including anisotropic thermal parameters for all non-hydrogen atoms was performed; the final results are given Tables 2-7. For these complexes a weighting scheme of the form $1 /\left[\sigma^{2}\left(F_{0}\right)+k\left(F_{\mathrm{o}}\right)^{2}\right], k=0.00002$ for complex 4 and $k=0$ for complexes 2,3 and 5-7, was used. All hydrogenatom parameters were calculated according to ideal geometries and were not refined. The structural analyses were made on a Microvax III computer using NRCVAX programs. ${ }^{22}$ Atomic scattering factors were taken from ref. 23.

Additional material available from the Cambridge Crystallographic Data Centre comprises $\mathrm{H}$-atom coordinates, thermal parameters and remaining bond lengths and angles.

\section{Results and Discussion}

Synthesis.-The ligand $N, N$-bis(pyrazol-1-ylmethyl)benzylamine (L), which possesses three nitrogen donor sites, was prepared using a modification of the literature method. ${ }^{21}$ Reactions of metal ions and ligand (L), which possesses two potential nitrogen donor sites from the pyrazolyl rings and one nitrogen donor site from benzylamine, result in the formation of complexes $\left[\mathrm{FeLCl}_{3}\right],\left[\mathrm{CoLCl}{ }_{2}\right],\left[\mathrm{CoL}(\mathrm{NCS})_{2}\right]$, $\left[\mathrm{NiL}(\mathrm{NCS})_{2}(\mathrm{MeOH})\right],\left[\mathrm{CuLCl}_{2}\right],\left[(\mathrm{CuLI})_{2}\right],\left[\mathrm{ZnLBr}_{2}\right]$, $\left[\mathrm{ZnL}(\mathrm{NCS})_{2}\right]$ and $\left[\mathrm{ZnLCl}_{2}\right]$

Molecular Structures of $\left[\mathrm{CoLCl}_{2}\right] 2$ and $\left[\mathrm{CoL}(\mathrm{NCS})_{2}\right] 3$. These structures are shown in Figs. 1 and 2; selected bond distances and angles are presented in Table 8.

In complex 2, the cobalt(II) ion is surrounded by two pyrazolyl nitrogen atoms at Co-N(1) 2.014(4) $\AA$ and $\mathrm{Co}-\mathrm{N}(3)$ $2.001(3) \AA$, and two chloride ions at $\mathrm{Co}-\mathrm{Cl}(1) 2.320$ (2) $\AA$ and $\mathrm{Co}-\mathrm{Cl}(2) 2.249$ (2) $\AA$. Some angles between co-ordination bonds of cobalt(II), which range from $95.9(1)$ to $123.7(1)^{\circ}$, deviate substantially from ideal tetrahedral angles. Bonding may also occur from Co to the amine $\mathrm{N}$ as the Co-N(3) distance is $2.510(4) \AA$. While the $\mathrm{Co}-\mathrm{N}($ amine) distance is longer than that found in $\left[\mathrm{CoL}_{2}{ }_{2}\right]\left[\mathrm{NO}_{3}\right]_{2}[2.229(5) \AA]\left[\mathrm{L}^{\prime}=\right.$ bis(1-pyrazolylmethyl)amine $],{ }^{24}$ in which the $\mathrm{Co}-\mathrm{N}$ bond is described as bonding, it is much shorter than that of $\left[\mathrm{Co}(\mathrm{bdmpab}) \mathrm{Cl}_{2}\right]$ $[3.854(2) \AA] \quad[$ bdmpab $=N, N$-bis(3,5-dimethylpyrazol-1-ylmethyl)aminobenzene],${ }^{5}$ in which the cobalt ion is described as having a distorted tetrahedral configuration. The geometry of 


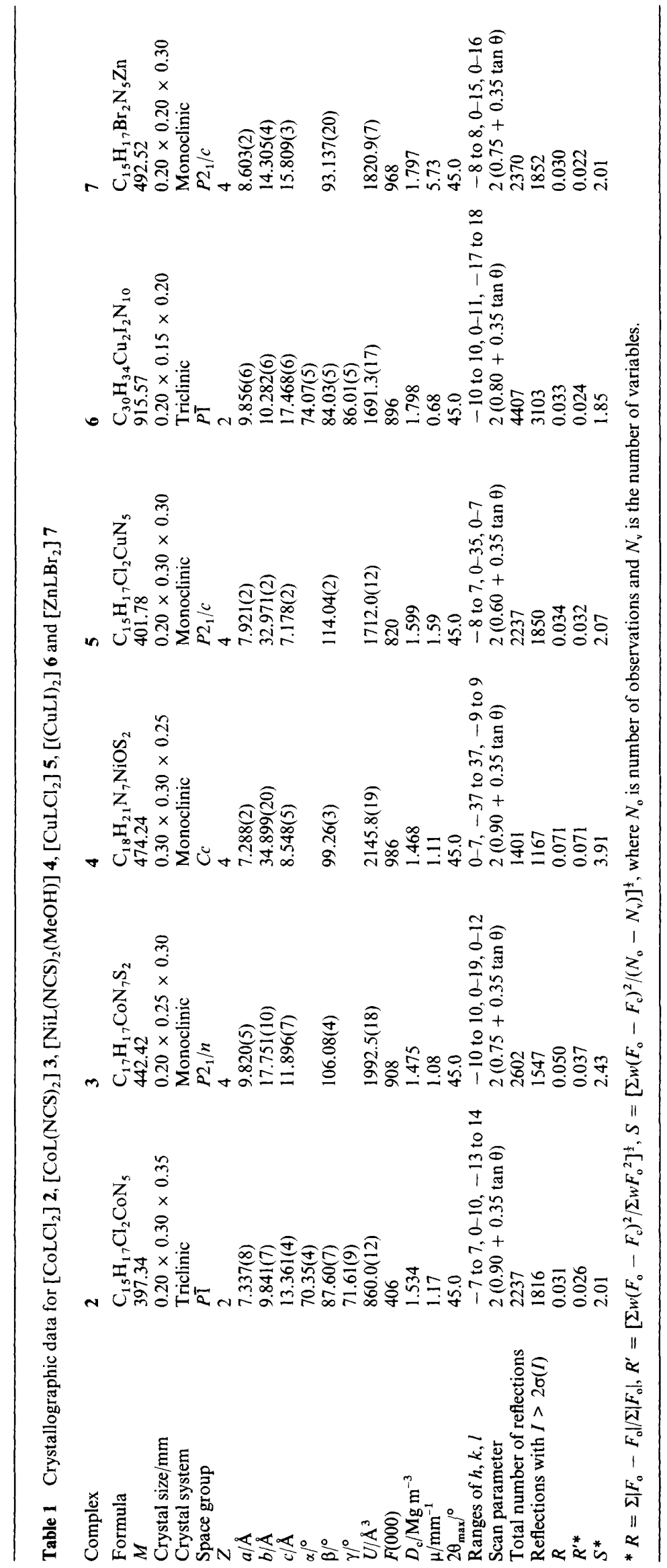


Table 2 Final atomic positional parameters of non-hydrogen atoms for $\left[\mathrm{CoLCl}_{2}\right] 2$

$\begin{array}{llll}\text { Atom } & x & y & z \\ \mathrm{Co} & 0.82481(8) & 0.93932(7) & 0.27389(5) \\ \mathrm{Cl}(1) & 0.63098(16) & 0.82377(13) & 0.39033(9) \\ \mathrm{Cl}(2) & 0.68576(18) & 1.02161(14) & 0.10823(9) \\ \mathrm{N}(1) & 1.0561(5) & 0.7506(4) & 0.3047(3) \\ \mathrm{N}(2) & 1.2306(5) & 0.7591(4) & 0.2714(3) \\ \mathrm{N}(3) & 0.8399(5) & 1.0780(4) & 0.35141(25) \\ \mathrm{N}(4) & 0.9952(5) & 1.1242(4) & 0.34911(25) \\ \mathrm{N}(5) & 1.1054(5) & 1.0308(4) & 0.20868(25) \\ \mathrm{C}(1) & 1.0910(6) & 0.6078(5) & 0.3699(3) \\ \mathrm{C}(2) & 1.2840(7) & 0.5249(5) & 0.3781(4) \\ \mathrm{C}(3) & 1.3686(6) & 0.6262(5) & 0.3144(4) \\ \mathrm{C}(4) & 0.7159(6) & 1.1540(5) & 0.4050(3) \\ \mathrm{C}(5) & 0.7908(7) & 1.2475(5) & 0.4363(3) \\ \mathrm{C}(6) & 0.9710(7) & 1.2242(5) & 0.4004(3) \\ \mathrm{C}(7) & 1.2408(6) & 0.8986(5) & 0.1894(3) \\ \mathrm{C}(8) & 1.1652(6) & 1.0512(5) & 0.3021(3) \\ \mathrm{C}(9) & 1.0828(6) & 1.1654(5) & 0.1088(3) \\ \mathrm{C}(10) & 0.9723(7) & 1.3147(5) & 0.1205(3) \\ \mathrm{C}(11) & 1.0687(7) & 1.4080(6) & 0.1341(3) \\ \mathrm{C}(12) & 0.9672(11) & 1.5499(7) & 0.1390(4) \\ \mathrm{C}(13) & 0.7717(11) & 1.5998(6) & 0.1293(5) \\ \mathrm{C}(14) & 0.6748(8) & 1.5083(6) & 0.1155(4) \\ \mathrm{C}(15) & 0.7734(7) & 1.3666(5) & 0.1107(4) \\ & & & \end{array}$

Table 3 Final atomic positional parameters of non-hydrogen atoms for $\left[\mathrm{CoL}(\mathrm{NCS})_{2}\right]^{3}$

\begin{tabular}{|c|c|c|c|}
\hline Atom & $x$ & $y$ & $z$ \\
\hline Co & $0.23660(13)$ & $0.16313(7)$ & $0.07281(10)$ \\
\hline$S(1)$ & $0.1433(3)$ & $0.10190(15)$ & $0.42918(21)$ \\
\hline $\mathbf{S}(2)$ & $0.7214(3)$ & $0.21199(16)$ & $0.21103(22)$ \\
\hline$N(1)$ & $0.2142(6)$ & $0.0927(3)$ & $-0.0664(5)$ \\
\hline$N(2)$ & $0.0952(6)$ & $0.0994(3)$ & $-0.1561(5)$ \\
\hline$N(3)$ & $0.2096(7)$ & $0.2725(4)$ & $0.0301(5)$ \\
\hline$N(4)$ & $0.0771(7)$ & $0.3002(3)$ & $-0.0144(5)$ \\
\hline$N(5)$ & $-0.0089(6)$ & $0.1751(3)$ & $-0.0409(5)$ \\
\hline$N(6)$ & $0.1706(6)$ & $0.1366(4)$ & $0.2091(5)$ \\
\hline$N(7)$ & $0.4494(7)$ & $0.1614(4)$ & $0.1364(6)$ \\
\hline$C(1)$ & $0.2820(9)$ & $0.0329(5)$ & $-0.0905(8)$ \\
\hline$C(2)$ & $0.2074(10)$ & $0.0017(5)$ & $-0.1948(7)$ \\
\hline$C(3)$ & $0.0926(10)$ & $0.0445(5)$ & $-0.2336(7)$ \\
\hline C(4) & $0.2941(9)$ & $0.3309(5)$ & $0.0249(7)$ \\
\hline$C(5)$ & $0.2173(11)$ & $0.3938(5)$ & $-0.0198(8)$ \\
\hline$C(6)$ & $0.0818(10)$ & $0.3731(5)$ & $-0.0430(8)$ \\
\hline$C(7)$ & $0.0083(8)$ & $0.1645(4)$ & $-0.1586(6)$ \\
\hline$C(8)$ & $-0.0436(9)$ & $0.2525(4)$ & $-0.0171(7)$ \\
\hline $\mathrm{C}(9)$ & $-0.1049(8)$ & $0.1189(4)$ & $-0.0113(6)$ \\
\hline$C(10)$ & $-0.2514(8)$ & $0.1143(4)$ & $-0.0994(6)$ \\
\hline$C(11)$ & $-0.2825(10)$ & $0.0563(5)$ & $-0.1771(8)$ \\
\hline$C(12)$ & $-0.4112(11)$ & $0.0536(6)$ & $-0.2577(8)$ \\
\hline$C(13)$ & $-0.5090(10)$ & $0.1086(6)$ & $-0.2596(8)$ \\
\hline$C(14)$ & $-0.4802(9)$ & $0.1654(6)$ & $-0.1817(8)$ \\
\hline$C(15)$ & $-0.3519(8)$ & $0.1684(5)$ & $-0.1009(7)$ \\
\hline $\mathrm{C}(16)$ & $0.1571(8)$ & $0.1219(4)$ & $0.3007(7)$ \\
\hline$C(17)$ & $0.5636(8)$ & $0.1828(5)$ & $0.1653(7)$ \\
\hline
\end{tabular}

the cobalt ion environment can thus also be described as intermediate between tetrahedral and trigonal bipyramidal.

The Co-N(pyrazole) distances [2.037(6) $\AA$ and 2.006(6) $\AA]$ of complex 3 are similar to those of complex 2 , but the Co-N(amine) distance $[2.425(6) \AA]$ is even smaller than that of complex $2[2.510(4) \AA]$ and the Co-N(amine) bonding interaction is thus stronger in complex 3 . Some of the angles between co-ordination bonds deviate significantly from ideal tetrahedral angles [range $99.2(3)-122.6(3)^{\circ}$ ] and as in complex 2 the geometry of the cobalt(II) environment can be described as intermediate between tetrahedral and trigonal bipyramidal. Two thiocyanate ions are bonded to the cobalt(II) ion [Co-N(6) 1.963(6) $\AA, \mathrm{Co}-\mathrm{N}(7) 2.016(7) \AA]$. The co-ordination angles of
Table 4 Final atomic positional parameters of non-hydrogen atoms for $\left[\mathrm{NiL}(\mathrm{NCS})_{2}(\mathrm{MeOH})\right] 4$

\begin{tabular}{|c|c|c|c|}
\hline Atom & $x$ & $y$ & $z$ \\
\hline $\mathrm{Ni}$ & 0.47916 & $0.89657(7)$ & 0.72123 \\
\hline$S(1)$ & $0.7430(8)$ & $0.81424(17)$ & $0.3772(7)$ \\
\hline$S(2)$ & $-0.0661(8)$ & $0.94743(25)$ & $0.4180(9)$ \\
\hline $\mathrm{N}(1)$ & $0.3244(20)$ & $0.8482(4)$ & $0.7414(18)$ \\
\hline$N(2)$ & $0.4063(19)$ & $0.8215(4)$ & $0.8354(18)$ \\
\hline$N(3)$ & $0.4136(19)$ & $0.9238(5)$ & $0.9130(17)$ \\
\hline$N(4)$ & $0.5154(20)$ & $0.9147(5)$ & $1.0541(19)$ \\
\hline$N(5)$ & $0.6557(19)$ & $0.8667(4)$ & $0.9296(17)$ \\
\hline$C(1)$ & $0.1874(24)$ & $0.8297(6)$ & $0.6453(22)$ \\
\hline$C(2)$ & $0.189(3)$ & $0.7929(7)$ & $0.685(3)$ \\
\hline$C(3)$ & $0.331(3)$ & $0.7881(6)$ & $0.807(3)$ \\
\hline $\mathrm{C}(4)$ & $0.278(3)$ & $0.9465(5)$ & $0.9579(24)$ \\
\hline$C(5)$ & $0.292(3)$ & $0.9495(6)$ & $1.121(3)$ \\
\hline$C(6)$ & $0.449(3)$ & $0.9291(6)$ & $1.1877(22)$ \\
\hline$C(7)$ & $0.5399(24)$ & $0.8335(6)$ & $0.9759(23)$ \\
\hline$C(8)$ & $0.6830(25)$ & $0.8937(5)$ & $1.0526(23)$ \\
\hline$C(9)$ & $0.8304(23)$ & $0.8509(5)$ & $0.8770(21)$ \\
\hline$C(10)$ & $0.9703(24)$ & $0.8311(5)$ & $1.0113(22)$ \\
\hline$C(11)$ & $0.982(3)$ & $0.7930(6)$ & $1.0196(24)$ \\
\hline$C(12)$ & $1.112(3)$ & $0.7743(7)$ & $1.137(3)$ \\
\hline$C(13)$ & $1.223(3)$ & $0.7990(6)$ & $1.2444(24)$ \\
\hline $\mathrm{C}(14)$ & $1.209(3)$ & $0.8364(6)$ & $1.2350(22)$ \\
\hline$C(15)$ & $1.084(3)$ & $0.854 \mathrm{I}(6)$ & $1.123(3)$ \\
\hline$N(6)$ & $0.5949(19)$ & $0.8685(5)$ & $0.5494(17)$ \\
\hline$C(16)$ & $0.6573(22)$ & $0.8452(5)$ & $0.4767(20)$ \\
\hline $\mathrm{N}(7)$ & $0.2801(23)$ & $0.9241(6)$ & $0.5732(20)$ \\
\hline$C(17)$ & $0.141(3)$ & $0.9330(6)$ & $0.5058(21)$ \\
\hline $\mathrm{O}$ & $0.6868(18)$ & $0.9389(3)$ & $0.7083(17)$ \\
\hline$C(18)$ & $0.653(4)$ & $0.9754(6)$ & $0.716(3)$ \\
\hline
\end{tabular}

Table 5 Final atomic positional parameters of non-hydrogen atoms for $\left[\mathrm{CuLCl}_{2}\right] 5$

\begin{tabular}{llll} 
Atom & \multicolumn{1}{l}{$y$} & $y$ & \multicolumn{1}{l}{} \\
$\mathrm{Cu}$ & $0.02720(8)$ & $0.145876(19)$ & $0.02825(9)$ \\
$\mathrm{Cl}(1)$ & $0.16681(18)$ & $0.20172(4)$ & $-0.08887(19)$ \\
$\mathrm{Cl}(2)$ & $-0.19276(19)$ & $0.11333(5)$ & $-0.23392(21)$ \\
$\mathrm{N}(1)$ & $-0.1363(6)$ & $0.18018(12)$ & $0.1075(6)$ \\
$\mathrm{N}(2)$ & $-0.0487(5)$ & $0.19916(12)$ & $0.2877(6)$ \\
$\mathrm{N}(3)$ & $0.2352(5)$ & $0.10952(12)$ & $0.0643(6)$ \\
$\mathrm{N}(4)$ & $0.3890(5)$ & $0.11698(12)$ & $0.2345(6)$ \\
$\mathrm{N}(5)$ & $0.1960(5)$ & $0.15359(11)$ & $0.3534(5)$ \\
$\mathrm{C}(1)$ & $-0.3153(7)$ & $0.19049(17)$ & $0.0430(8)$ \\
$\mathrm{C}(2)$ & $-0.3376(7)$ & $0.21667(17)$ & $0.1810(10)$ \\
$\mathrm{C}(3)$ & $-0.1659(8)$ & $0.22144(16)$ & $0.3359(9)$ \\
$\mathrm{C}(4)$ & $0.2753(7)$ & $0.07798(16)$ & $-0.0231(7)$ \\
$\mathrm{C}(5)$ & $0.4546(8)$ & $0.06588(18)$ & $0.0873(8)$ \\
$\mathrm{C}(6)$ & $0.5237(7)$ & $0.09105(18)$ & $0.2527(8)$ \\
$\mathrm{C}(7)$ & $0.1529(6)$ & $0.19554(15)$ & $0.3902(7)$ \\
$\mathrm{C}(8)$ & $0.3853(6)$ & $0.15063(15)$ & $0.3646(7)$ \\
$\mathrm{C}(9)$ & $0.1605(7)$ & $0.12569(15)$ & $0.4951(7)$ \\
$\mathrm{C}(10)$ & $0.1474(7)$ & $0.08143(15)$ & $0.4344(8)$ \\
$\mathrm{C}(11)$ & $0.2927(8)$ & $0.05507(18)$ & $0.5298(8)$ \\
$\mathrm{C}(12)$ & $0.2746(9)$ & $0.01476(20)$ & $0.4738(11)$ \\
$\mathrm{C}(13)$ & $0.1148(11)$ & $0.00098(20)$ & $0.3193(12)$ \\
$\mathrm{C}(14)$ & $-0.0294(9)$ & $0.02683(20)$ & $0.2240(10)$ \\
$\mathrm{C}(15)$ & $-0.0134(7)$ & $0.06682(17)$ & $0.2816(8)$ \\
& & &
\end{tabular}

the thiocyanates to the metal ion deviates from linearity to a moderate degree $\left[167.7(6)\right.$ and $\left.159.4(7)^{\circ}\right]$.

Molecular Structure of $\left[\mathrm{NiL}(\mathrm{NCS})_{2}(\mathrm{MeOH})\right]$ 4.--This structure is shown in Fig. 3; selected bond distances and angles are presented in Table 8.

The nickel(II) ion is six-coordinate with the ligand bound in a facial manner [Ni-N(1) 2.052(15), Ni-N(3) 2.018(15) and $\mathrm{Ni}-\mathrm{N}(5) 2.276(14) \AA]$. Two isocyanate ions $[\mathrm{Ni}-\mathrm{N}(6) 2.056(15)$ and $\mathrm{Ni}-(7) 2.009(15) \AA]$ and one oxygen from methanol [Ni-O $2.130(13) \AA]$ occupy the remaining facial site. Distortion from 
Table 6 Final atomic positional parameters of non-hydrogen atoms for $\left[(\mathrm{CuLI})_{2}\right] 6$

\begin{tabular}{|c|c|c|c|c|c|c|c|}
\hline Atom & $x$ & $y$ & $z$ & Atom & $x$ & $y$ & $z$ \\
\hline I(1) & $0.59142(5)$ & $0.97561(5)$ & $0.13344(3)$ & $C(14)$ & $1.4754(8)$ & $0.3742(8)$ & $0.1191(5)$ \\
\hline I(2) & $0.83654(5)$ & $0.78311(6)$ & $0.33130(3)$ & $C(15)$ & $1.4011(8)$ & $0.4944(7)$ & $0.0960(4)$ \\
\hline $\mathrm{Cu}(1)$ & $0.82134(10)$ & $0.84418(10)$ & $0.17059(6)$ & $N(6)$ & $0.4487(6)$ & $0.7598(6)$ & $0.3480(3)$ \\
\hline $\mathrm{Cu}(2)$ & $0.59510(10)$ & $0.89637(10)$ & $0.29114(6)$ & $\mathrm{N}(7)$ & $0.3118(6)$ & $0.7766(6)$ & $0.3449(3)$ \\
\hline$N(1)$ & $0.9855(5)$ & $0.9701(6)$ & $0.1262(3)$ & $N(8)$ & $0.5573(5)$ & $1.0428(6)$ & $0.3499(3)$ \\
\hline$N(2)$ & $1.1028(5)$ & $0.9497(5)$ & $0.0806(3)$ & $N(9)$ & $0.4419(6)$ & $1.1175(6)$ & $0.355 \mathrm{~S}(3)$ \\
\hline $\mathrm{N}(3)$ & $0.8527(5)$ & $0.6744(6)$ & $0.1337(3)$ & $\mathrm{N}(10)$ & $0.2375(6)$ & $1.0154(6)$ & $0.3285(3)$ \\
\hline $\mathrm{N}(4)$ & $0.9713(5)$ & $0.5978(5)$ & $0.1382(3)$ & $C(21)$ & $0.4676(8)$ & $0.6359(8)$ & $0.3973(4)$ \\
\hline $\mathrm{N}(5)$ & $1.1806(5)$ & $0.7108(5)$ & $0.0930(3)$ & $\mathrm{C}(22)$ & $0.3428(8)$ & $0.5753(7)$ & $0.4248(4)$ \\
\hline$C(1)$ & $1.0066(8)$ & $1.0826(7)$ & $0.1460(4)$ & $\mathrm{C}(23)$ & $0.2463(8)$ & $0.6679(8)$ & $0.3913(5)$ \\
\hline$C(2)$ & $1.1312(8)$ & $1.1333(7)$ & $0.1151(4)$ & $C(24)$ & $0.6333(7)$ & $1.0733(7)$ & $0.3995(4)$ \\
\hline $\mathrm{C}(3)$ & $1.1903(7)$ & $1.0466(7)$ & $0.0746(4)$ & $\mathrm{C}(25)$ & $0.5667(8)$ & $1.1646(8)$ & $0.4373(4)$ \\
\hline$C(4)$ & $0.7729(7)$ & $0.6099(8)$ & $0.0997(5)$ & $C(26)$ & $0.4457(8)$ & $1.1922(7)$ & $0.4071(4)$ \\
\hline$C(5)$ & $0.8395(8)$ & $0.4997(9)$ & $0.0815(5)$ & $\mathrm{C}(27)$ & $0.2492(7)$ & 0.903 l(7) & $0.2957(4)$ \\
\hline $\mathrm{C}(6)$ & $0.9651(8)$ & $0.4947(7)$ & $0.1063(5)$ & $\mathrm{C}(28)$ & $0.3337(7)$ & $1.1203(7)$ & $0.3025(4)$ \\
\hline$C(7)$ & $1.1216(7)$ & $0.8344(7)$ & $0.0457(4)$ & $C(29)$ & $0.1470(7)$ & $1.0098(7)$ & $0.4007(4)$ \\
\hline $\mathrm{C}(8)$ & $1.0900(7)$ & $0.6437(7)$ & $0.1632(4)$ & $\mathrm{C}(30)$ & $0.0667(7)$ & $1.1417(7)$ & $0.3952(4)$ \\
\hline$C(9)$ & $1.3182(7)$ & $0.7230(7)$ & $0.1133(4)$ & $C(31)$ & $-0.0190(8)$ & $1.1873(9)$ & $0.3357(5)$ \\
\hline$C(10)$ & $1.3928(6)$ & $0.5877(7)$ & $0.1410(4)$ & $\mathrm{C}(32)$ & $-0.0865(10)$ & $1.3077(10)$ & $0.3276(6)$ \\
\hline$C(11)$ & $1.4599(7)$ & $0.5563(7)$ & $0.2085(4)$ & $C(33)$ & $-0.0775(10)$ & $1.3874(9)$ & $0.3774(7)$ \\
\hline$C(12)$ & $1.5356(8)$ & $0.4364(9)$ & $0.2314(4)$ & $C(34)$ & $0.0059(10)$ & $1.3372(10)$ & $0.4382(6)$ \\
\hline$C(13)$ & $1.5444(8)$ & $0.3456(8)$ & $0.1859(5)$ & $C(35)$ & $0.0782(8)$ & $1.2158(9)$ & $0.4472(5)$ \\
\hline
\end{tabular}

Table 7 Final atomic positional parameters of non-hydrogen atoms for $\left[\mathrm{ZnLBr}_{2}\right] 7$

\begin{tabular}{llll} 
Atom & $x$ & $y$ & \multicolumn{1}{l}{} \\
$\mathrm{Zn}$ & $0.97060(7)$ & $0.24734(4)$ & $0.07105(4)$ \\
$\mathrm{Br}(1)$ & $0.82921(7)$ & $0.39143(4)$ & $0.06984(4)$ \\
$\mathrm{Br}(2)$ & $0.85416(7)$ & $0.13082(4)$ & $-0.02297(4)$ \\
$\mathrm{N}(1)$ & $0.9653(5)$ & $0.1971(3)$ & $0.1898(3)$ \\
$\mathrm{N}(2)$ & $1.0373(5)$ & $0.1174(3)$ & $0.21861(24)$ \\
$\mathrm{N}(3)$ & $1.1969(4)$ & $0.2757(3)$ & $0.03100(25)$ \\
$\mathrm{N}(4)$ & $1.3231(5)$ & $0.2147(3)$ & $0.0223(3)$ \\
$\mathrm{N}(5)$ & $1.2797(5)$ & $0.1140(3)$ & $0.13837(25)$ \\
$\mathrm{C}(1)$ & $0.8785(6)$ & $0.2284(4)$ & $0.2532(3)$ \\
$\mathrm{C}(2)$ & $0.8921(7)$ & $0.1686(4)$ & $0.3214(3)$ \\
$\mathrm{C}(3)$ & $0.9961(7)$ & $0.0985(4)$ & $0.2979(3)$ \\
$\mathrm{C}(4)$ & $1.2458(6)$ & $0.3563(4)$ & $-0.0021(3)$ \\
$\mathrm{C}(5)$ & $1.4019(7)$ & $0.3457(4)$ & $-0.0326(4)$ \\
$\mathrm{C}(6)$ & $1.4485(6)$ & $0.2553(4)$ & $-0.0164(3)$ \\
$\mathrm{C}(7)$ & $1.1405(6)$ & $0.0605(3)$ & $0.1640(3)$ \\
$\mathrm{C}(8)$ & $1.3080(6)$ & $0.1179(3)$ & $0.0508(3)$ \\
$\mathrm{C}(9)$ & $1.4254(6)$ & $0.1225(4)$ & $0.1984(3)$ \\
$\mathrm{C}(10)$ & $1.5219(6)$ & $0.0327(3)$ & $0.2133(3)$ \\
$\mathrm{C}(11)$ & $1.4860(6)$ & $-0.0263(4)$ & $0.2771(3)$ \\
$\mathrm{C}(12)$ & $1.5713(7)$ & $-0.1085(4)$ & $0.2905(4)$ \\
$\mathrm{C}(13)$ & $1.6975(7)$ & $-0.1318(4)$ & $0.2407(4)$ \\
$\mathrm{C}(14)$ & $1.7367(6)$ & $-0.0728(4)$ & $0.1770(4)$ \\
$\mathrm{C}(15)$ & $1.6498(6)$ & $0.0094(4)$ & $0.1627(3)$ \\
& & & \\
\hline
\end{tabular}

octahedral symmetry is fairly substantial considering that the relevant angles lie between $76.0(3)$ and $96.6(7)^{\circ}$. The $\mathrm{Ni}-\mathrm{N}$ (amine) bond which is trans to the $\mathrm{NCS}^{-}$is longer than that found in $\left[\mathrm{NiL}_{2}^{\prime}\right]\left[\mathrm{BF}_{4}\right]_{2}[2.182(9) \AA] .{ }^{25}$ The coordination angles of the thiocyanates to the metal ion deviate from linearity $\left[163.4(16)\right.$ and $\left.162.4(19)^{\circ}\right]$.

Molecular Structure of $\left[\mathrm{CuLCl}_{2}\right]$ 5.-This structure is shown in Fig. 4; selected bond distances and angles are presented in Table 8.

In complex 5 , the copper(II) ion is co-ordinated by a benzylamine nitrogen atom at $\mathrm{Cu}-\mathrm{N}(5) 2.182(4) \AA$, and two pyrazolyl nitrogen atoms at $\mathrm{Cu}-\mathrm{N}(1) 1.970(4)$ and $\mathrm{Cu}-\mathrm{N}(3)$ $1.968(4) \AA$, and two chloride ions at $\mathrm{Cu}-\mathrm{Cl}(1) 2.465(2)$ and $\mathrm{Cu}-\mathrm{Cl}(2) 2.248(2) \AA$. Addison et al. ${ }^{26}$ defined the $\tau$ parameter ( $\tau=0$, square-pyramidal geometry; $\tau=1$, trigonal-bipyramidal geometry) to distinguish between these geometries. The $\tau$ value for complex 5 is 0.10 , indicating that the co-ordination

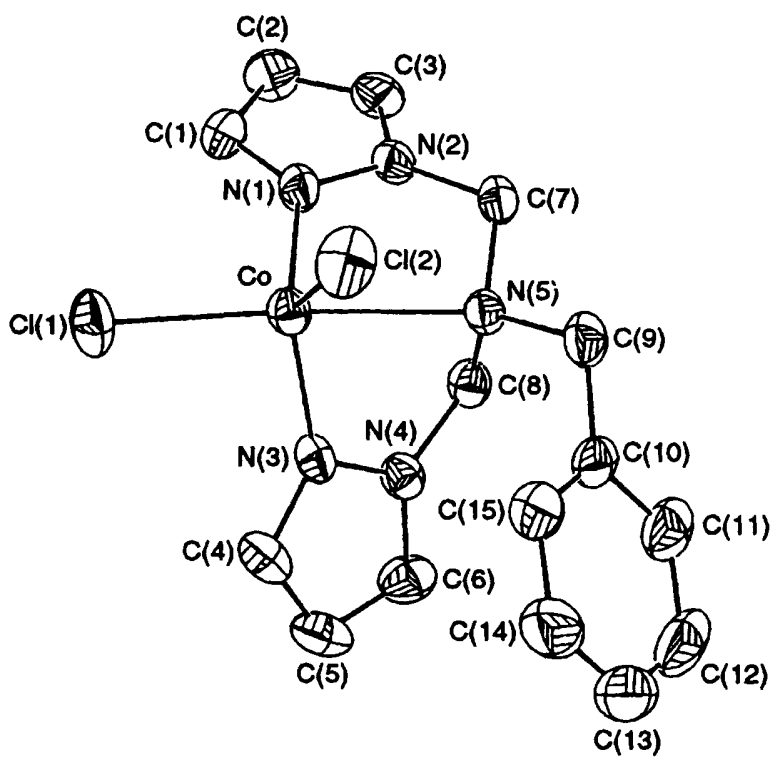

Fig. 1 Molecular structure of $\left[\mathrm{CoLCl}_{2}\right] 2$ with $50 \%$ probability ellipsoids for non-hydrogen atoms

sphere of copper(II) is better described as distorted square pyramidal rather than distorted trigonal bipyramidal. The geometry and the $\mathrm{Cu}-\mathrm{N}$ distances are similar to those of $\left[\mathrm{Cu}(\mathrm{ddae}) \mathrm{Cl}_{2}\right]$ \{ddae = bis[2-(3,5-dimethylpyrazol-1-yl)ethyl)]ethylamine $\}^{27}$ in which the ligand forms six-membered chelate rings upon co-ordination. The basal plane involves $\mathrm{N}(1), \mathrm{N}(5), \mathrm{N}(3)$ and $\mathrm{Cl}(2)$ while $\mathrm{Cl}(1)$ occupies the apical position; the $\mathrm{Cu}^{\text {II }}$ ion lies $0.418(2) \AA$ above the basal plane.

Molecular Structure of $\left[(\mathrm{CuLI})_{2}\right]$ 6. - This structure is shown in Fig. 5; selected bond distances and angles are presented in Table 8 .

The copper(I) ion is surrounded by two nitrogen atoms at $\mathrm{Cu}(1)-\mathrm{N}(1) 2.092(6)$ and $\mathrm{Cu}(1)-\mathrm{N}(3) 2.015(6) \AA$ and by two bridging iodide ions at $\mathrm{Cu}(1)-\mathrm{I}(1) 2.604(2)$ and $\mathrm{Cu}(1)-\mathrm{I}(2)$ $2.721(2) \AA$. Two copper(I) ions and two bridging iodide ions form a planar $\mathrm{Cu}_{2} \mathrm{I}_{2}$ four-membered ring. The $\mathrm{Cu}(1) \cdots \mathrm{Cu}(2)$ distance at $3.028(2) \AA$, is larger than that in other known 
Table 8 Selected bond distances $(\AA)$ and angles $\left({ }^{\circ}\right)$ for $\left[\mathrm{CoLCl}_{2}\right] 2,\left[\mathrm{CoL}(\mathrm{NCS})_{2}\right] 3,\left[\mathrm{NiL}(\mathrm{NCS})_{2}(\mathrm{MeOH})\right] 4,\left[\mathrm{CuLCl}{ }_{2}\right] 5,\left[(\mathrm{CuLI})_{2}\right] 6$ and $\left[\mathrm{ZnLBr}_{2}\right]$ 7. For the atomic numbering see Figs. 1-6

\begin{tabular}{|c|c|c|c|c|c|c|c|}
\hline \multicolumn{4}{|l|}{$\left[\mathrm{CoLCl}_{2}\right] 2$} & \multicolumn{4}{|l|}{$\left[\mathrm{CuLCl}_{2}\right] 5$} \\
\hline $\mathrm{Co}-\mathrm{N}(1)$ & $2.014(4)$ & $\mathrm{Co}-\mathrm{Cl}(1)$ & $2.320(2)$ & $\mathrm{Cu}-\mathrm{N}(1)$ & $1.970(4)$ & $\mathrm{Cu}-\mathrm{Cl}(1)$ & $2.465(2)$ \\
\hline Co-N(3) & $2.001(3)$ & $\mathrm{Co}-\mathrm{Cl}(2)$ & $2.249(2)$ & $\mathrm{Cu}-\mathrm{N}(3)$ & $1.968(4)$ & $\mathrm{Cu}-\mathrm{Cl}(2)$ & $2.248(2)$ \\
\hline $\mathrm{Co}-\mathrm{N}(5)$ & $2.510(4)$ & & & $\mathrm{Cu}-\mathrm{N}(5)$ & $2.182(4)$ & & \\
\hline $\mathrm{N}(1)-\mathrm{Co}-\mathrm{N}(3)$ & $113.98(15)$ & $\mathrm{Cl}(1)-\mathrm{Co}-\mathrm{N}(3)$ & $96.02(11)$ & $\mathrm{N}(1)-\mathrm{Cu}-\mathrm{N}(3)$ & $157.77(16)$ & $\mathrm{Cl}(1)-\mathrm{Cu}-\mathrm{N}(3)$ & $91.83(12)$ \\
\hline $\mathrm{N}(1)-\mathrm{Co}-\mathrm{N}(5)$ & $74.12(14)$ & $\mathrm{Cl}(1)-\mathrm{Co}-\mathrm{N}(5)$ & $159.66(9)$ & $\mathrm{N}(1)-\mathrm{Cu}-\mathrm{N}(5)$ & $79.03(15)$ & $\mathrm{Cl}(1)-\mathrm{Cu}-\mathrm{N}(5)$ & $96.26(10)$ \\
\hline $\mathrm{N}(3)-\mathrm{Co}-\mathrm{N}(5)$ & $72.98(23)$ & $\mathrm{Cl}(2)-\mathrm{Co}-\mathrm{N}(1)$ & $113.36(13)$ & $\mathrm{N}(3)-\mathrm{Cu}-\mathrm{N}(5)$ & $79.52(15)$ & $\mathrm{Cl}(2)-\mathrm{Cu}-\mathrm{N}(1)$ & $98.00(13)$ \\
\hline $\mathrm{Cl}(1)-\mathrm{Co}-\mathrm{Cl}(2)$ & $107.57(8)$ & $\mathrm{Cl}(2)-\mathrm{Co}-\mathrm{N}(3)$ & $123.74(11)$ & $\mathrm{Cl}(1)-\mathrm{Cu}-\mathrm{Cl}(2)$ & $111.92(6)$ & $\mathrm{Cl}(2)-\mathrm{Cu}-\mathrm{N}(3)$ & $98.12(12)$ \\
\hline $\mathrm{Cl}(1)-\mathrm{Co}-\mathrm{N}(1)$ & $95.90(12)$ & $\mathrm{Cl}(2)-\mathrm{Co}-\mathrm{N}(5)$ & $92.72(10)$ & $\mathrm{Cl}(1)-\mathrm{Cu}-\mathrm{N}(1)$ & $96.23(13)$ & $\mathrm{Cl}(2)-\mathrm{Cu}-\mathrm{N}(5)$ & $151.81(11)$ \\
\hline \multicolumn{4}{|l|}{$\left[\mathrm{CoL}(\mathrm{NCS})_{2}\right] 3$} & \multicolumn{4}{|l|}{$\left[(\mathrm{CuLI})_{2}\right] 6$} \\
\hline Co--N(1) & $2.037(6)$ & $\mathrm{Co}-\mathrm{N}(3)$ & $2.006(6)$ & $\mathrm{Cu}(1)-\mathrm{N}(1)$ & $2.092(6)$ & $\mathrm{Cu}(1)-\mathrm{I}(2)$ & $2.721(2)$ \\
\hline $\mathrm{Co}-\mathrm{N}(5)$ & $2.425(6)$ & $\mathrm{Co}-\mathrm{N}(6)$ & $1.963(6)$ & $\mathrm{Cu}(1)-\mathrm{N}(3)$ & $2.015(6)$ & $\mathrm{Cu}(2)-\mathrm{I}(1)$ & $2.653(2)$ \\
\hline \multirow[t]{2}{*}{$\mathrm{Co}-\mathrm{N}(7)$} & $2.016(7)$ & & & $\mathrm{Cu}(2)-\mathrm{N}(6)$ & $2.064(6)$ & $\mathrm{Cu}(2)-\mathrm{I}(2)$ & $2.650(2)$ \\
\hline & & & & $\mathrm{Cu}(2)-\mathrm{N}(8)$ & $2.037(6)$ & $\mathrm{Cu}(1) \cdots \mathrm{Cu}(2)$ & $3.028(2)$ \\
\hline $\mathrm{N}(1)-\mathrm{Co}-\mathrm{N}(3)$ & $114.3(3)$ & $\mathrm{N}(1)-\mathrm{Co}-\mathrm{N}(5)$ & $74.54(22)$ & $\mathrm{Cu}(1)-\mathrm{I}(1)$ & $2.604(2)$ & & \\
\hline $\mathrm{N}(1)-\mathrm{Co}-\mathrm{N}(6)$ & $122.6(3)$ & $\mathrm{N}(1)-\mathrm{Co}-\mathrm{N}(7)$ & $99.4(3)$ & & & & \\
\hline $\mathrm{N}(3)-\mathrm{Co}-\mathrm{N}(5)$ & $74.49(22)$ & $\mathrm{N}(3)-\mathrm{Co}-\mathrm{N}(6)$ & $113.0(3)$ & $\mathrm{Cu}(1)-\mathrm{I}(1)-\mathrm{Cu}(2)$ & $70.34(6)$ & $\mathrm{N}(1)-\mathrm{Cu}(1)-\mathrm{N}(3)$ & $109.67(22)$ \\
\hline $\mathrm{N}(3)-\mathrm{Co}-\mathrm{N}(7)$ & $99.2(3)$ & $\mathrm{N}(5)-\mathrm{Co}-\mathrm{N}(6)$ & $88.68(23)$ & $\mathrm{Cu}(1)-\mathrm{I}(2)-\mathrm{Cu}(2)$ & $68.62(6)$ & $\mathrm{I}(1)-\mathrm{Cu}(2)-\mathrm{I}(2)$ & $110.63(6)$ \\
\hline $\mathrm{N}(5)-\mathrm{Co}-\mathrm{N}(7)$ & $167.86(24)$ & $\mathrm{N}(6)-\mathrm{Co}-\mathrm{N}(7)$ & $103.4(3)$ & $\mathrm{I}(1)-\mathrm{Cu}(1)-\mathrm{I}(2)$ & $109.93(6)$ & $\mathrm{I}(1)-\mathrm{Cu}(2)-\mathrm{N}(6)$ & $113.83(16)$ \\
\hline \multirow[t]{2}{*}{$\mathrm{Co}-\mathrm{N}(6)-\mathrm{C}(16)$} & $167.7(6)$ & $\mathrm{Co}-\mathrm{N}(7)-\mathrm{C}(17)$ & $159.4(7)$ & $\mathrm{I}(1)-\mathrm{Cu}(1)-\mathrm{N}(1)$ & $110.54(16)$ & $\mathrm{I}(2)-\mathrm{Cu}(2)-\mathrm{N}(8)$ & $116.57(16)$ \\
\hline & & & & $\mathrm{I}(1)-\mathrm{Cu}(1)-\mathrm{N}(3)$ & $114.00(16)$ & $\mathrm{I}(2)-\mathrm{Cu}(2)-\mathrm{N}(6)$ & $107.73(16)$ \\
\hline \multicolumn{4}{|c|}{$\left[\mathrm{NiL}(\mathrm{NCS})_{2}(\mathrm{MeOH})\right] 4$} & $\mathbf{I}(2)-\mathrm{Cu}(1)-\mathrm{N}(1)$ & $102.31(16)$ & $\mathbf{I}(2)-\mathrm{Cu}-(2)-\mathbf{N}(8)$ & $105.61(16)$ \\
\hline $\mathrm{Ni}-\mathrm{N}(1)$ & $2.052(15)$ & $\mathrm{Ni}-\mathrm{N}(3)$ & $2.018(15)$ & $\mathrm{I}(2)-\mathrm{Cu}(1)-\mathrm{N}(3)$ & $109.75(17)$ & $\mathrm{N}(6)-\mathrm{Cu}(2)-\mathrm{N}(8)$ & $101.67(23)$ \\
\hline $\mathrm{Ni}-\mathrm{N}(5)$ & $2.276(14)$ & $\mathrm{Ni}-\mathrm{N}(6)$ & $2.056(15)$ & & & & \\
\hline \multirow[t]{2}{*}{$\mathrm{Ni}-\mathrm{N}(7)$} & $2.009(15)$ & $\mathrm{Ni}-\mathrm{O}$ & $2.130(13)$ & {$\left[\mathrm{ZnLBr}_{2}\right] 7$} & & & \\
\hline & & & & $\mathrm{Zn}-\mathrm{Br}(1)$ & $2.355(1)$ & $\mathrm{Zn}-\operatorname{Br}(2)$ & $2.391(1)$ \\
\hline $\mathrm{N}(1)-\mathrm{Ni}-\mathrm{N}(3)$ & $96.5(6)$ & $\mathrm{N}(1)-\mathrm{Ni}-\mathrm{N}(3)$ & $78.9(6)$ & $\mathrm{Zn}-\mathrm{N}(1)$ & $2.013(4)$ & $\mathrm{Zn}-\mathrm{N}(3)$ & $2.005(4)$ \\
\hline $\mathrm{N}(1)-\mathrm{Ni}-\mathrm{N}(6)$ & $87.8(6)$ & $\mathrm{N}(1)-\mathrm{Ni}-\mathrm{N}(7)$ & $95.4(7)$ & $\mathrm{Zn}-\mathrm{N}(5)$ & $3.270(4)$ & & \\
\hline $\mathrm{N}(1)-\mathrm{Ni}-\mathrm{O}$ & $168.3(5)$ & $\mathrm{N}(3)-\mathrm{Ni}-\mathrm{N}(5)$ & $76.0(5)$ & & & & \\
\hline $\mathrm{N}(3)-\mathrm{Ni}-\mathrm{N}(6)$ & $169.4(5)$ & $\mathrm{N}(3)-\mathrm{Ni}-\mathrm{N}(7)$ & $92.6(7)$ & $\operatorname{Br}(1)-\mathrm{Zn}-\mathrm{Br}(2)$ & $115.57(4)$ & $\mathrm{Br}(1)-\mathrm{Zn}-\mathrm{N}(1)$ & $106.57(11)$ \\
\hline $\mathrm{N}(3)-\mathrm{Ni}-\mathrm{O}$ & $88.8(6)$ & $\mathrm{N}(5)-\mathrm{Ni}-\mathrm{N}(6)$ & $95.5(6)$ & $\mathrm{Br}(1)-\mathrm{Zn}-\mathrm{N}(3)$ & $105.58(11)$ & $\mathrm{Br}(2)-\mathrm{Zn}-\mathrm{N}(1)$ & $107.64(11)$ \\
\hline $\mathrm{N}(5)-\mathrm{Ni}-\mathrm{N}(7)$ & $166.4(6)$ & $\mathrm{N}(5)-\mathrm{Ni}-\mathrm{O}$ & $92.4(5)$ & $\mathrm{Br}(2)-\mathrm{Zn}-\mathrm{N}(3)$ & $106.15(11)$ & $\mathrm{N}(1)-\mathrm{Zn}-\mathrm{N}(3)$ & $115.66(16)$ \\
\hline $\mathrm{N}(6)-\mathrm{Ni}-\mathrm{N}(7)$ & $96.6(7)$ & $\mathrm{N}(6)-\mathrm{Ni}-\mathrm{O}$ & $85.2(6)$ & & & & \\
\hline $\mathrm{N}(7)-\mathrm{Ni}-\mathrm{O}$ & $94.8(7)$ & $\mathrm{Ni}-\mathrm{N}(6)-\mathrm{C}(16)$ & $163.4(19)$ & & & & \\
\hline $\mathrm{Ni}-\mathrm{N}(7)-\mathrm{C}(17)$ & $162.4(19)$ & & & & & & \\
\hline
\end{tabular}

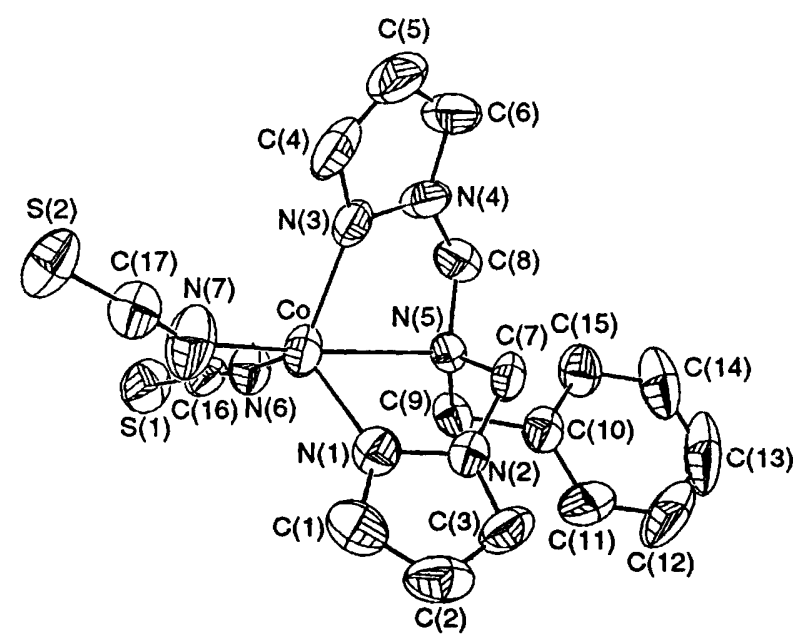

Fig. 2 Molecular structure of $\left[\mathrm{CoL}(\mathrm{NCS})_{2}\right] 3$ with $50 \%$ probability ellipsoids for non-hydrogen atoms

structural examples of isolated planar $\mathrm{Cu}_{2} \mathrm{X}_{2}$ rhombohedra: $\left[\left\{\mathrm{CuI}\left(\mathrm{AsPPh}_{3}\right)(\mathrm{MeCN})_{2}\right\}_{2}\right][2.779(1) \AA]^{28}$ and $\left[\left\{\mathrm{Cu}\left(\mathrm{Pr} \mathrm{P}^{\mathrm{i}} \mathrm{HN}-\right.\right.\right.$ $\left.\left.\left.\mathrm{CH}_{2} \mathrm{CH}_{2} \mathrm{NHPr}^{\mathrm{i}}\right) \mathrm{I}\right\}_{2}\right] \quad[2.733(1) \AA],{ }^{29}$ but a little smaller than that found in $\left[\left\{\mathrm{CuI}\left(\mathrm{C}_{9} \mathrm{H}_{7} \mathrm{~N}\right)\right\}_{2}\right]\left(\mathrm{C}_{9} \mathrm{H}_{7} \mathrm{~N}=\right.$ quinoline $)$ $\left[\begin{array}{ll}3.364(5) & \AA\end{array}\right] .{ }^{30}$ The $\mathrm{Cu}(1)-\mathrm{I}(1)-\mathrm{Cu}(2)$ bridging angle is $70.34(6)^{\circ}$ and the $\mathrm{I}(1)-\mathrm{Cu}(1)-\mathrm{I}(2)$ angle is $109.93(6)^{\circ}$. The benzylamine nitrogen-copper distances are 3.950(6) and 3.702(6) $\AA$ which are too long to be regarded as bonding. The

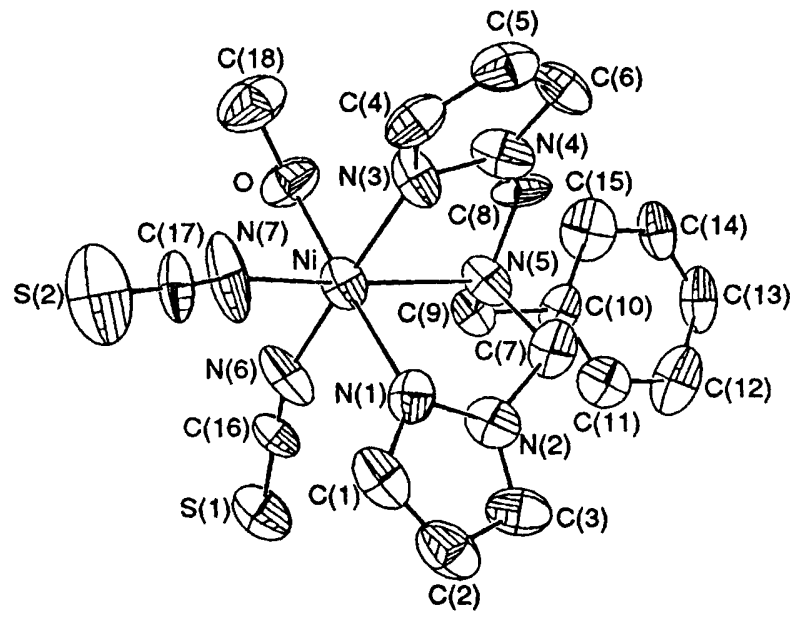

Fig. 3 Molecular structure of $\left[\mathrm{NiL}(\mathrm{NCS})_{2}(\mathrm{MeOH})\right] 4$ with $50 \%$ probability ellipsoids for non-hydrogen atoms

co-ordination of copper(I) can be described as distorted tetrahedral with angles from $102.31(16)$ to $110.54(16)^{\circ}$ for $\mathrm{Cu}(1)$ and from $101.67(23)$ to $116.57(16)^{\circ}$ for $\mathrm{Cu}(2)$.

The structure of complex 6 is very similar to that of $[\mathrm{Cu}-$ (aebd)I] [aebd $=N, N$-bis(3,5-dimethylpyrazol-1-ylmethyl)aminoethane $]^{31}$ which is mononuclear and complex 6 can be regarded as being formed from two copper(I) moieties like $[\mathrm{Cu}(\mathrm{aebd}) \mathrm{I}]$. However as for $\left[\{\mathrm{Cu}(\mathrm{aebp}) \mathrm{Cl}\}_{2}\right][$ aebp $=N, N-$ bis(pyrazol-1-ylmethyl)aminoethane $]^{32}$ formation of a bridge 
occurs in complex 6 because there is no ligand steric hindrance in contrast to aebd where methyl groups prevent association of the $[\mathrm{Cu}($ aebd $) \mathrm{I}]$ moieties.

Molecular Structure of $\left[\mathrm{ZnLBr}_{2}\right]$ 7.--This structure is shown in Fig. 6; selected bond distances and angles are presented in Table 8

In complex 7, the zinc(II) ion is surrounded by two pyrazolyl nitrogen atoms at $\mathrm{Zn}-\mathrm{N}(1) 2.013(4)$ and $\mathrm{Zn}-\mathrm{N}$ (3) 2.005(4) $\AA$, and two bromide ions at $\mathrm{Zn}-\mathrm{Br}(1) 2.355(1)$ and $\mathrm{Zn}-\mathrm{Br}(2)$ 2.391(1) $\AA$. The benzylamine nitrogen-zinc distance is $3.270(4)$ $\AA[\mathrm{Zn}-\mathrm{N}(5)]$ which is too long to be considered as a bonding interaction. The co-ordination sphere of zinc(II) can be described as only slightly distorted from tetrahedral with angles ranging from $105.58(11)$ to $115.66(16)^{\circ}$

Comparison of the structures of complexes 2-7 indicates that $N, N$-bis(pyrazol-1-ylmethyl)benzylamine is a flexible ligand. The cobalt(II) $\left(\mathrm{d}^{7}\right)$ and zinc(II) $\left(\mathrm{d}^{10}\right)$ ions favour tetrahadral geometry. In complexes 2 and 3 the amine rotates toward the Co ion, but away from the $\mathrm{Zn}$ ion in complex 7 . This results in $\mathrm{Co}-\mathrm{N}$ (amine) distances being shorter than that of $\mathrm{Zn}-\mathrm{N}$ (amine). The shorter $\mathrm{Co}-\mathrm{N}$ distances in $\mathbf{2}$ and $\mathbf{3}$ leads to some interaction between Co and N(amine) and to deviation from tetrahedral toward trigonal-bipyramidal geometry. The $\mathrm{Ni}-\mathrm{N}$ (amine) bond in complex 4 can be described as bonding for nickel(II) (d $\left.{ }^{8}\right)$ which prefers octahedral geometry. In the $\mathrm{d}^{10}$ copper(I) and zinc(II) complexes 6 and 7, the metal ion can be regarded as tetrahedral and the ligand is bidentate. For the octahedral

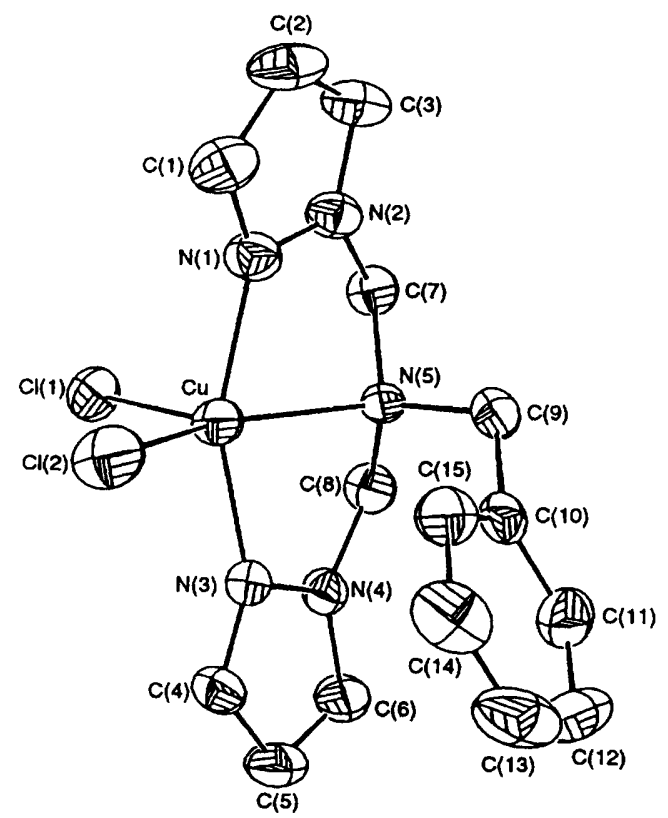

Fig. 4 Molecular structure of $\left[\mathrm{CuLCl}_{2}\right] 5$ with $50 \%$ probability ellipsoids for non-hydrogen atoms
nickel(II) complex 4 and square-pyramidal copper(II) in complex 5 the ligand is tridentate and the $\mathrm{M}-\mathrm{N}$ (amine) distances are within the range observed for other complexes. ${ }^{16-20}$ In all the complexes, the pyrazole rings are planar and the methyl carbons are coplanar with the respective pyrazole ring; the pyrazole rings are not mutually coplanar and there is no stacking between the aromatic rings with crystal packing determined by normal van der Waals contacts.

Spectroscopy.-In the IR spectra the $\mathrm{C}-\mathrm{H}$ and $\mathrm{C}=\mathrm{N}$ stretching vibrations of the pyrazolyl groups of the complexes are similar to those of the free ligand at 3107 and $1507 \mathrm{~cm}^{-1}$. There are new strong features at 2077 and $2060 \mathrm{~cm}^{-1}$ (complex 3), $2088 \mathrm{~cm}^{-1}$ (complex 4) and $2083 \mathrm{~cm}^{-1}$ (complex 8) arising from $\mathrm{N}$-coordinated $\mathrm{NCS}^{-}$groups. Complex 4 shows a broad absorption at $3334 \mathrm{~cm}^{-1}$ due to $v(\mathrm{OH})$ from co-ordinated methanol.

The far-IR spectra of $\left[\mathrm{CoLCl}_{2}\right] 2$ shows bands at 308 and $278 \mathrm{~cm}^{-1}$ characteristic of $v(\mathrm{Co}-\mathrm{Cl})^{5,33,34}$ while $v(\mathrm{Cu}-\mathrm{Cl})$ in $\left[\mathrm{CuLCl}_{2}\right] 5$ is located as one strong band at $258 \mathrm{~cm}^{-1}$. The chloro and bromo zinc complexes show very similar IR spectra except for absorptions at 284 and $223 \mathrm{~cm}^{-1}$ due to $v(\mathrm{Zn}-\mathrm{Cl})$ and $v(\mathrm{Zn}-\mathrm{Br})$ respectively; these values being characteristic for a tetrahedral structure. ${ }^{33}$ The ligand-field spectra of complexes 2-5 are reported in Table 9.

The ${ }^{1} \mathrm{H}$ NMR signals of complex 6 showed a large positive chemical shift of the methylene bridge protons at $\delta 5.20$, pyrazolyl $\mathrm{C}^{5} \mathrm{H}$ at $\delta 7.75$ and pyrazolyl $\mathrm{C}^{3} \mathrm{H}$ at $\delta 7.88$ relative to that of the free ligand $(\delta 4.95,7.45$ and 7.53$)$. The ${ }^{1} \mathrm{H}$ NMR signals of the zinc(II) complexes 7-9 also showed large positive chemical shifts of the methylene bridge protons at $\delta 5.07,5.05$ and 5.09, pyrazolyl $\mathrm{C}^{5} \mathrm{H}$ at $\delta 7.54,7.53$ and 7.55 , and pyrazolyl $\mathrm{C}^{3} \mathrm{H}$ at $\delta 7.76,7.76$ and 7.77 relative to that of the ligand $(\delta 4.95$, 7.45 and 7.53). Upon co-ordination most of the pyrazolyl proton signals were merged and broadened.

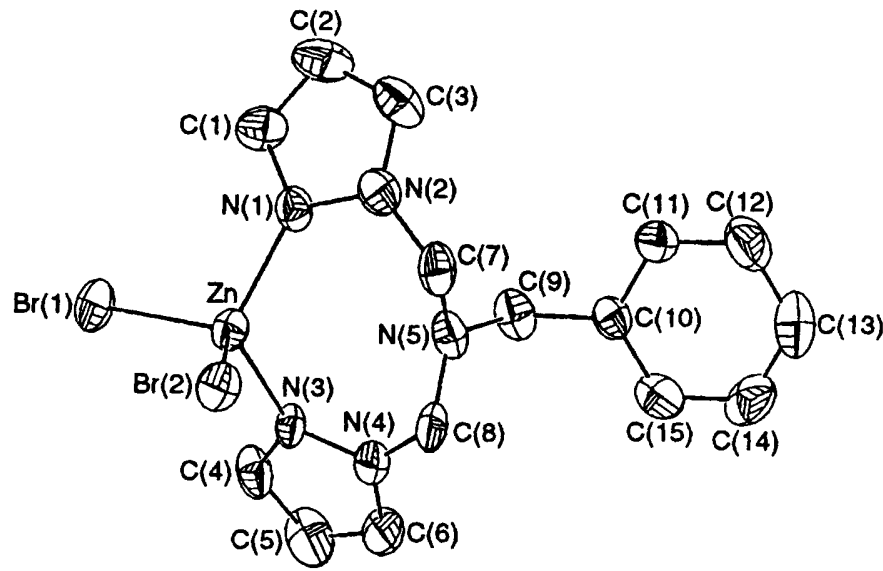

Fig. 6 Molecular structure of $\left[\mathrm{ZnLBr}_{2}\right] 7$ with $50 \%$ probability ellipsoids for non-hydrogen atoms

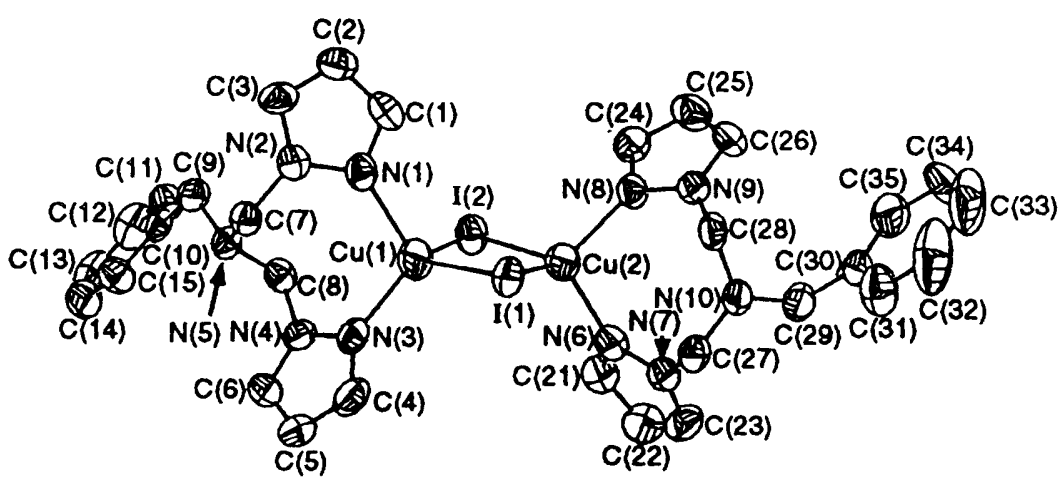

Fig. 5 Molecular structure of $\left[(\mathrm{CuLI})_{2}\right] 6$ with $50 \%$ probability ellipsoids for non-hydrogen atoms 
Table 9 Electronic absorption maxima of co-ordination compounds of $\mathrm{L}$

$\begin{array}{ll}\text { Compound } & \text { Ligand-field bands }\left(\mathrm{cm}^{-1}\right) \\ {\left[\mathrm{CoLCl}{ }_{2}\right]} & 17667,16667 \\ {\left[\mathrm{CoL}(\mathrm{NCS})_{2}\right]} & 17123 \\ {\left[\mathrm{NiL}(\mathrm{NCS})_{2}(\mathrm{MeOH})\right]} & 27778,13405,12771 \\ {\left[\mathrm{CuLCl}{ }_{2}\right]} & 25906,16233,12987\end{array}$

The X-band EPR spectra and magnetic moments of the polycrystalline complexes were measured at $298 \mathrm{~K}$. The EPR spectrum of complex 5 is similar to that of $[\{\mathrm{Cu}(\mathrm{bmdhp})$ $\left.\left.\left(\mathrm{H}_{2} \mathrm{O}\right)\left(\mathrm{ClO}_{4}\right)\right\}_{2}\right]^{26} \quad[\mathrm{bmdhp}=1,7$-bis $(N$-methylbenzimidazol2-yl)-2,6-dithiaheptane] and shows an axial signal. ${ }^{35}$ The $g$ values $\left(g_{\|}=2.243, g_{\perp}=2.130\right)$ of complex 5 are consistent with a square-pyramidal co-ordination sphere for copper(II) and the single unpaired electron is located in an essentially $\mathrm{d}_{x^{2}-y^{2}}$ orbital. The $g_{\text {iso }}$ value is 2.150 in MeOH-MeCN solution at $77 \mathrm{~K}$. The EPR spectra of complexes 2 and 3 at $298 \mathrm{~K}$ show no signal, but do show broad isotropic signals at $77 \mathrm{~K}$, with $g$ values of 3.307 and 3.552 . The magnetic moments $\left(\mu_{\text {eff }}\right)$ of complexes $\mathbf{1}-\mathbf{5}$ are $5.98,4.55,4.40,3.19$ and $1.94 \mu_{B}$, respectively. These values indicate that the octahedral iron(III) ion ( $\mathrm{d}^{5}$ configuration) has high spin $\left(S=\frac{5}{2}\right)$ for complex 1 and the tetrahedral cobalt(II) ion ( $\mathrm{d}^{7}$ configuration) has high spin $\left(S=\frac{3}{2}\right)^{36}$ for complexes 2 and 3 .

\section{Acknowledgements}

We thank the National Science Council of the Republic of China for support.

\section{References}

1 F. S. Keij, R. A. de Graff, J. G. Haasnoot and J. Reedijk, J. Chem. Soc., Dalton Trans., 1984, 2093.

2 R. Prins, P. J. W. L. Birker, J. G. Haasnoot, G. C. Verschoor and J. Reedijk, Inorg. Chem., 1985, 24, 4128.

3 M. Angaroni, G. A. Ardizzoia, T. Beringhelli, G. La Monica, D. Gatteschi, N. Masciocchi and M. Moret, J. Chem. Soc., Dalton Trans., 1990, 3305.

4 F. S. Keij, J. G. Haasnoot, A. J. Oosterling, J. Reedijk, C. J. O'Connor, J. H. Zhang and A. L. Spek, Inorg. Chim. Acta, 1991, $181,185$.

5 E. Bouwman, W. L. Driessen and J. Reedijk, Inorg. Chem., 1985, 24, 4730; H. L. Blonk, W. L. Driessen and J. Reedijk, J. Chem. Soc., Dalton Trans., 1985, 1699; J. B. J. Veldhuis, W. L. Driessen and J. Reedijk, J. Chem. Soc., Dalton Trans., 1986, 537.

6 S. M. Morehouse, A. Polychronopoulou and G. L. B. Williams, Inorg. Chem., 1980, 19, 3558.

7 A. C. van Steenbergen, E. Bouwman, R. A. G. de Graaff, W. L. Driessen, J. Reedijk and P. Zanello, J. Chem. Soc., Dalton Trans., 1990, 3175.

8 E. S. Raper and W. Clegg, Inorg. Chim. Acta, 1991, 180, 239.

9 S. M. Wang, P. J. Huang, H. Chang, C. Y. Cheng, S. L. Wang and N. C. Li, Inorg. Chim. Acta, 1991, 182, 109.

10 M. Hadded, D. N. Hendrickson, J. P. Cannady, R. S. Drago and D. S. Bieksza, J. Am. Chem. Soc., 1979, 101, 898.
11 F. Abraham, M. Lagrenee, S. Sueur, B. Mernari and C. Bremard, J. Chem. Soc., Dalton Trans., 1991, 1443.

12 S. Kitagawa, M. Munakata and T. Tanimura, Inorg. Chem., 1992, 31, 1714.

13 K. D. Karlin, R. W. Cruse and Y. Gultneh, J. Chem. Soc., Chem. Commun., 1987, 599; J. E. Pate, R. W. Cruse, K. D. Karlin and E. I. Solomon, J. Am. Chem. Soc., 1987, 110, 2624; K. D. Karlin, R. W. Cruse, Y. Gultneh, A. Farooq, J. C. Hayes and J. Zubieta, J. Am. Chem. Soc., 1987, 109, 2668; K. D. Karlin, M. S. Haka, R. W. Cruse, G. J. Meyer, A. Farooq, Y. Gultneh, J. C. Hayes and J. Zubieta, J. Am. Chem. Soc., 1988, 109, 1196; N. J. Blackburn, R. W. Strange, A. Farooq, M. S. Haka and K. D. Karlin, J. Am. Chem. Soc., 1988, 110, 4263; K. D. Karlin, P. Ghosh, R. W. Cruse, A. Farooq, Y. Gultneh, R. R. Jacobson, N. J. Blackburn R. W. Strange and J. Zubieta, J. Am. Chem. Soc., 1988, $110,6769$.

14 S. M. Nelson, F. Esho, A. Lavery and M. G. B. Drew, J. Am. Chem. Soc., 1983, 105, 5693 .

15 N. Kitajima, T. Koda, S. Hashimoto, T. Kitagawa and Y. Morooka, J. Am. Chem. Soc., 1991, 113, 5664; N. Kitajima, K. Fujisawa, C. Fujimoto, Y. Moro-oka, S. Hashimoto, T. Kitagawa, K. Toriumi, K. Tatsumi and A. Nakamura, J. Am. Chem. Soc., 1992, 114, 1277.

16 T. N. Sorrell, M. L. Garrity and D. J. Ellis, Inorg. Chim. Acta, 1989 , 166, 71; T. N. Sorrell and V. A. Vankai, Inorg. Chem., 1990, 29, 1687; T. N. Sorrell, V. A. Vankai and M. L. Garrity, Inorg. Chem., 1991, 30, 207.

17 C. T. Chen, W. K. Chang, S. C. Sheu, G. H. Lee, T. I. Ho, Y. C. Lin and Y. Wang, J. Chem. Soc, Dalton Trans., 1991, 1569.

18 W. K. Chang, S. C. Sheu, G. H. Lee, Y. Wang, T. I. Ho and Y. C. Lin, J. Chem. Soc., Dalton Trans., 1993, 687.

19 W. K. Chang, G. H. Lee, Y. Wang, Y. O. Su, T. I. Ho and Y. C. Lin, Inorg. Chim. Acta, 1994, 223, 139.

20 W. K. Chang, Ph.D. Thesis, National Taiwan University, 1994.

21 W. L. Driessen, Recl. Trav. Chim. Pays-Bas, 1982, 101, 41; 1. Dvoretzky and G. H. Richter, J. Org. Chem., 1950, 15, 1285.

22 E. J. Gabe, Y. Le Page, P. S. White and F. L. Lee, Acta Crystallogr., Sect. $A, 1973,43, \mathrm{~S} 294$

23 International Tables for X-Ray Crystallography, Kynoch Press, Birmingham, 1974, vol. 4.

24 W. L. Driessen, W. G. R. Wiesmeijer, M. Schipper-Zablotskaja, R. A. G. de Graff and J. Reedijk, Inorg. Chim. Acta, 1989, 162, 233.

25 A. L. Spek, A. J. M. Duisenberg, W. L. Driessen and W. G. R. Wiesmeijer, Acta Crystallogr., Sect. C, 1988, 44, 623.

26 A. W. Addison, T. N. Rao, J. Reedijk, J. van Rijn and G. C. Verschoor, J. Chem. Soc., Dalton Trans., 1984, 1349.

27 W. L. Driessen, R. A. G. de Graaff, F. J. Parlevliet, J. Reedijk and R. M. de Vos, Inorg. Chim. Acta, 1994, 216, 43.

28 M. R. Churchill and J. R. Missert, Inorg. Chem., 1981, 20, 619.

29 N. P. Rath, E. M. Holt and K. Tanimura, J. Chem. Soc., Dalton Trans., 1986, 2303.

30 D. A. Haitko, J. Coord. Chem., 1984, 13, 119.

31 Y. C. M. Pennings, W. L. Driessen and J. Reedijk, Acta Crystallogr., Sect. C, 1988, 44, 2095.

32 Y. C. M. Pennings, W. L. Driessen and J. Reedijk, Polyhedron, 1988, $7,2583$.

$33 \mathrm{~J}$. R. Ferraro, Low-Frequency Vibrations of Inorganic and Coordination Compounds, Plenum Press, New York, 1971.

34 G. J. van driel, W. L. Driessen and J. Reedijk, Inorg. Chem., 1985, 24, 2919.

35 B. J. Hathaway and D. E. Billing, Coord. Chem. Rev., 1970, 5, 143.

36 B. N. Figgis and R. S. Nyholm, J. Chem. Soc., 1959, 338; B. J. Hathaway and D. G. Holah, J. Chem. Soc., 1964, 2400.

Received 16th June 1995; Paper 5/03919C 